University of Nebraska - Lincoln

DigitalCommons@University of Nebraska - Lincoln

1987

\title{
Asymmetries in Mating Preferences between Species: Female Swordtails Prefer Heterospecific Males
}

\author{
Michael J. Ryan \\ University of Texas, mryan@mail.utexas.edu \\ William E. Wagner Jr. \\ University of Nebraska-Lincoln, wwagner1@unl.edu
}

Follow this and additional works at: https://digitalcommons.unl.edu/bioscifacpub

Ryan, Michael J. and Wagner, William E. Jr., "Asymmetries in Mating Preferences between Species:

Female Swordtails Prefer Heterospecific Males" (1987). Faculty Publications in the Biological Sciences.

239.

https://digitalcommons.unl.edu/bioscifacpub/239

This Article is brought to you for free and open access by the Papers in the Biological Sciences at DigitalCommons@University of Nebraska - Lincoln. It has been accepted for inclusion in Faculty Publications in the Biological Sciences by an authorized administrator of DigitalCommons@University of Nebraska - Lincoln. 
Asymmetries in Mating Preferences between Species: Female Swordtails Prefer Heterospecific Males

Michael J. Ryan and William E. Wagner, Jr.

Department of Zoology, University of Texas, Austin, Texas

\begin{abstract}
In male swordtails (Xiphophorus nigrensis) there are three size classes that derive from allelic variation at the pituitary locus on the $\mathrm{Y}$ chromosome. Progeny analysis and preference tests suggest that females prefer to mate with larger males. In the closely related $X$. pygmaeus, there is no allelic variation at this locus; this species consists of males similar in size only to smaller $X$. nigrensis males. In addition to being smaller than most $X$. nigrensis males, these $X$. pygmaeus males also lack both the swordtail and a major component of the courtship display common in most $X$. nigrensis males. Usually, female $X$. pygmaeus prefer to mate with heterospecific males rather than conspecifics, regardless of body size and the presence of a swordtail. However, the smallest $X$. nigrensis males lack the same courtship component as do the $X$. pygmaeus males, and in this comparison female $X$. pygmaeus show no preference. Although sexual selection, through its action on divergence of courtship displays, has been implicated as a factor leading to speciation, in this case sexual selection could lead to the congealing of gene pools between heterospecifics.
\end{abstract}

In many species, mating attempts by a male can be successful only with the cooperation of the female. Female choice can have important effects at two levels: it can result in mating with conspecifics instead of heterospecifics (interspecies discrimination), and it can enhance the mating success of some conspecific males relative to others (intraspecies discrimination).

Mate choice usually is hierarchical; intraspecies discrimination, or sexual selection, acts within the constraints of interspecies discrimination, or species recognition. Furthermore, researchers have suggested that divergence of courtship signals under sexual selection can lead to speciation as individuals from different populations fail to recognize one another as conspecific (1). We report that females of two species of swordtail (Xiphophorus, Poecili- idae family) exhibit preference for mates and that in one species females prefer heterospecific males to their own conspecifics. This heterospecific preference results from lack of a courtship display component in conspecific males combined with similar preference by females of both species for full courtship display. These data suggest that sexual selection can not only have a diversifying effect, as suggested above (1), but can also override species recognition and potentially act as a congealing force between closely related species.

Many species of Xiphophorus are characterized by considerable variation in body size (2). Much of this variation is heritable [for example, greater than 90\% for the Rio Choy, Mexico, population of $X$. nigrensis (3)], and it results from allelic variation at the pituitary
(P) locus on the $\mathrm{Y}$ chromosome (2). In species with a greater number of alleles at the P locus, body size is distributed continuously, but in $X$. nigrensis from the Rio Choy, there are only three alternative alleles at the $P$ locus, which results in three discrete body size classes (2). Swordtails have internal fertilization, females choose their mates, and less preferred males attempt to force copulation with females $(4,5)$. Paternity analysis of progeny from females collected in the field demonstrates a mating advantage for larger males in $X$. nigrensis (6). Laboratory tests reveal female mating preferences for these larger males that are consistent with the greater mating success of larger males in nature (6).

We wanted to determine if this preference for large males in X. nigrensis could be generalized to closely related species. If so, females of closely related species that did not have large males should prefer to mate with heterospecifics. This preference would constitute a unique example of mate choice overriding considerations of species recognition, and would demonstrate sexual selection that potentially gives rise to heterospecific preference. Xiphophorus pygmaeus and $X$. nigrensis are allopatric and closely related (7). In the former species, the P locus also influences male body size, but only the allele that results in smaller males is present (3).

Therefore, we tested the hypothesis of heterospecific preference by giving female $X$. pygmaeus a choice between their own conspecific and a larger $X$. nigrensis male. Xiphophorus pygmaeus contains gold and blue males; thus in these initial tests females were tested with either a gold [26 mm standard 
Table 1 . The amount of time spent by female X. nigrensis and X. pygmaeus with courting males of different sizes and species. Abbreviations: Mean s, mean number of seconds; $\mathrm{C}$, conspecific (relative to the female tested); $\mathbf{C b}$, blue conspecific; $\mathbf{C g}$, gold conspecific; $\mathbf{H}$, heterospecific (relative to the female tested); Hns, heterospeciflc with swordtail removed; $t$, paired $t$ test statistic; $\mathrm{df}$, degrees of freedom; and $P$, probability.

\begin{tabular}{llrrr}
\multicolumn{2}{c}{ Test males, mean s (SE) } & & & \\
Conspecific & \multicolumn{1}{c}{$\begin{array}{c}\text { Heterospecific } \\
\text { Female Xiphophorus pygmaeus }\end{array}$} & & df & $P$ \\
Cg, 26 mm; 302 s (39.2) & H, 37 mm; 744 s (63.9) & 10.9 & 10 & $<0.001$ \\
Cb, 26 mm; 285 s (24.7) & H, 37 mm; 764 s (24.4) & 4.3 & 10 & $<0.01$ \\
Cb, 26 mm; 351 s (39.2) & H, 26 mm; 740 s (46.9) & 4.6 & 9 & $<0.01$ \\
Cb, 26 mm; 331 s (81.3) & Hns, 26 mm; 801 s (85.5) & 2.8 & 9 & $<0.05$ \\
Cb, 26 mm; 571 s (65.7) & H, 24 mm; 503 s (72.6) & 0.5 & 9 & $>0.25$ \\
Cb, 23 mm; 533 s (51.7) & H, 23 mm; 555 s (64.2) & 0.2 & 9 & $>0.25$ \\
& Female Xiphophorus nigrensis & & \\
C, 26 mm; 711 s (44.4) & H, 26 mm; 412 s (47.6) 3.3 & 9 & $<0.01$
\end{tabular}

length (SL)] or a blue conspecific male (26 mm SL) against a larger heterospecific (37 mm SL). Eleven females were tested in four trials: twice with a blue conspecific-heterospecific pair, and twice with a gold conspecific-heterospecific pair. The testing apparatus was an aquarium ( 45 by 90 by $41 \mathrm{~cm}$ ) that was divided into five equal sections. The sections at each end were separated from the three central sections by plexiglass. A male was placed in each of these end sections. The plexiglass partition ensured that females were exposed only to visual cues. An opaque cylinder (11 $\mathrm{cm}$ in diameter) was placed in the middle section; a female was placed in the cylinder and allowed to acclimate for 10 minutes. After the female had acclimated, the cylinder was removed and the female had the opportunity to be in one of the three sections-either the center section or one of the sections adjacent to a male. We recorded the amount of time the female spent in each section during the following 10 minutes. After the first trial, the female was returned to the cylinder, the males were switched between the end sections of the tank, and the trial was repeated.

This switching controlled for side biases. In all trials described below, males and females attempted to court one another with species-typical behaviors through the glass partition; thus this test appears to measure courting preference. We used a two-tailed paired $t$ test to evaluate the null hypothesis that the amount of time a female spent with each male was equal (8). In both comparisons females preferred heterospecific males (Table 1). In subsequent tests, only blue X. pygmaeus males were used.
The above results suggest that $X$. pygmaeus females, like $X$. nigrensis females, prefer larger males, even though these larger males are heterospecifics and greatly exceed the size of any male $X$. pygmaeus. However, other differences are apparent between males of these two species: differences in general body form and the lack of the swordtail in X. pygmaeus. Also, Franck (4) reported that X. pygmaeus males do not exhibit the elaborate sexual display associated with courtship-the most conspicuous component of the full courtship display. In this respect, X. pygmaeus males resemble small $X$. nigrensis males and differ from larger $X$. nigrensis males. To test the hypothesis that body size was the cause of the heterospecific preference, we presented ten female $X$. pygmaeus with a choice between a heterospecific and conspecific of the same size (26 mm SL). Females showed a preference for the heterospecific in this comparison in which body size differences were eliminated (Table 1). In the same comparison, female $X$. nigrensis similarly preferred the male $X$. nigrensis, its conspecific (Table 1). Although the males were the same size, the male $X$. nigrensis possessed a swordtail.

To test the importance of this variable, we surgically removed the swordtail and again tested female $X$. pygmaeus; again, the $X$. pygmaeus females preferred the $X$. nigrensis, the heterospecific. Thus neither body size nor swordtail alone accounts for the heterospecific preference.

As noted above, males also differ in their courtship behavior. The hypothesis that the display accounts for the female preference would indicate that when female $X$. pygmaeus are given a choice between a conspecific and a small male $X$. nigrensis, which also fails to exhibit the sexual display, the heterospecific preference would be eliminated.

This was the case when X. pygmaeus and small $X$. nigrensis males were the same size and when $X$. nigrensis males were smaller (Table 1$)$.

These results demonstrate that, in most comparisons, $X$. pygmaeus females prefer heterospecifics. This preference seems to be based on the presence or absence of full courtship behavior, since it is eliminated when small, noncourting $X$. nigrensis males are tested.

One possible explanation for the heterospecific preference is that the female preference for full courtship was shared by an ancestor common to $X$. nigrensis and $X$. pygmaeus, and it has been retained in females of both species even though this courtship trait is lacking in male X. pygmaeus. Mating asymmetries also have been reported in Drosophila. Unlike those in X. pygmaeus, however, the mating asymmetries in Drosophila are characterized by random mating between conspecifics and heterospecifics rather than preference for heterospecifics as we have demonstrated here (9).

In Drosophila, female preference combined with the lack of a courtship component also is suggested to be responsible for the mating asymmetry, but the efficacy of female choice and differences in courtship behaviors have not been demonstrated. Similar results are found among stickleback populations.

McPhail (10) showed that female sticklebacks from populations with either red or black nuptial colors both preferred red males. The taxonomic status of the red and black male populations of sticklebacks is not clear, but they are generally considered the same species (10).

Our study of interspecific mating preferences in Xiphophorus demonstrates that females prefer to mate with heterospecific males rather than their own conspecifics.

Recent theoretical models, discussions, and data suggest that sexual selection on courtship traits generated by female choice can result in speciation as conspecifics in adjacent populations fail to respond to each other as members of the same species (1). In contradistinction, sexual selection could act as a con- 
gealing force for the species pair of $X$. nigrensis and X. pygmaeus. These species readily hybridize in the laboratory with no obvious deleterious effects on hybrid offspring. If these allopatric species were to become sympatric, preference of $X$. pygmaeus females for $\mathrm{X}$. nigrensis males could result in extensive introgression, and perhaps the convergence of these two species into one cohesive gene pool.

\section{References and Notes}

1. R. Lande, Evolution 36, 213 (1982); R. Thornhill and J. Alcock, The Evolution of Insect Mating Systems (Harvard Univ. Press, Cambridge, MA, 1983); M.J. West Eberhard, Q. Rev. Biol. 58, 155 (1983); W.J. Domincy, in Evolution of Fish Species Flocks, A.A. Echelle and I. Kornfield, Eds. (Univ. of Maine Press, Orono, 1984), pp. 231-249; E. Mayr, ibid., pp. 3-11; R.D. Sage, P.V. Loiselle, P. Basasibwaki, A.C. Wilson, ibid., pp. 185-201; G.R. Smith and T. Todd, ibid., pp. 45-68; M.J. Ryan, Proc. Natl. Acad. Sci. U.S.A. 83, 1379 (1986).

2. K.D. Kallman, M.P. Schreibman, V. Borkoski, Science 181, 678 (1973); K. D. Kallman and V. Borkoski, Genetics 89, 79 (1978); K.D. Kallman, in Evolutionary Genetics of Fishes, B.J. Turner, Ed. (Plenum, New York, 1984), pp. 95-171.

3. Analysis by M.J. Ryan of unpublished data provided by K.D. Kallman.

4. D. Franck, Zool. Anz. 173, 315 (1964); Zool. Jb. Physiol. 71, 117 (1964); J. Hemens, Behav iour 27, 290 (1966); J.D. Nelson, thesis, University of California, Santa Cruz (1976).

5. M.J. Ryan and B. Causey, in preparation. 6. M.J. Ryan, D.K. Hews, W.E. Wagner, in preparation; K.D. Kallman (personal communication) demonstrated that small males reproduce at a rate much lower than that expected given their frequency in the population.

7. D.E. Rosen, Bull. Am. Mus. Nat. Hist. 162, 267 (1979); Nei's genetic distance between the species is 0.13 [D.C. Morizot and M.J. Siciliano, Genetics 102, 539 (1982)], which is greater than that found between some fish species in different genera [for example, Sage et al. (1)].

8.G.W.SnedecorandW.G.Cochran, Statistical Methods (Iowa State Univ. Press, Ames, 1967).

9. K.Y. Kaneshiro, Evolution 34, 437 (1981); L.V. Giddings and A.R. Templeton, Science 220, 372 (1983).

10. J.D. McPhail, J. Fish. Res. Board Can. 26, 3183 (1969); G.E.E. Moodie, Can. J. Zool. 50, 721 (1972).

11. We thank G. Barlow, J. Endler, M. Kirkpatrick, B. Sullivan, and M.J. West Eberhard for comments on the manuscript; C. Chusid and R. Stanford for assistance in the laboratory; D. Hews, K. Winemiller, and S. Weller for assistance in the field; and G. Santos for permission to work on his property. We thank K.D. Kallman and D. Morizot for their advice throughout this project. This work was supported by two Reeder fellowships and a University Research Institute (University of Texas) grant to M.J.R.

Manuscript received October 10, 1986; accepted February 12, 1987. 